\title{
Cognitive Processes Underlying Women's Risk Judgments: Associations With Sexual Victimization History and Rape Myth Acceptance
}

\author{
Elizabeth A. Yeater \\ University of New Mexico
}

\author{
Teresa A. Treat \\ Yale University
}

\author{
Richard J. Viken and Richard M. McFall \\ Indiana University
}

\begin{abstract}
Objective: This study evaluated the effects of sexual victimization history, rape myth acceptance, implicit attention, and recent learning on the cognitive processes underlying undergraduate women's explicit risk judgments. Method: Participants were 194 undergraduate women between 18 and 24 years of age. The sample was ethnically diverse and composed primarily of freshman, heterosexual, and single women. Stimuli were written vignettes describing social situations that varied on dimensions of sexual victimization risk and potential impact on women's popularity. Participants completed cognitive tasks assessing relative attention to victimization risk versus popularity impact, learning about either risk or popularity impact, and explicit classification of victimization risk. Participants then completed the Sexual Experiences Survey (SES) and the Rape Myth Acceptance Scale; SES responses were used to quantify the severity of victimization experiences. Results: More severe victimization history predicted use of higher thresholds for judging situations as risky, as well as lower sensitivity to risk and greater sensitivity to popularity impact when judging risk. Greater rape myth acceptance also predicted lower sensitivity to risk information. Higher relative attention to victimization risk predicted greater sensitivity to risk information when judging risk. Recent learning about either the risk or the popularity impact aspects of social situations modified sensitivity to risk versus popularity when making risk judgments. Conclusion: The study emphasizes the importance of distinguishing the threshold for judging situations as risky from sensitivity to risk-relevant information in understanding individual differences in women's risk judgments. Both processes may be important to consider when developing interventions to reduce women's risk for sexual victimization.
\end{abstract}

Keywords: sexual victimization, risk perception, rape myths, attention, learning

College women report high rates of sexual victimization. Approximately $50 \%$ of college women have experienced some form of sexual aggression, with $25 \%$ of those women experiencing an attempted or completed rape (Fisher, Cullen, \& Turner, 2000; Koss, Gidycz, \& Wisniewski, 1987). Not surprisingly, the effects of such victimization can be harmful and long-lasting. One of the more serious of these is that, once victimized, women are at increased risk for future victimization (Gidycz, Coble, Latham, \& Layman, 1993; Messman-Moore \& Long, 2003). To date, the mechanisms responsible for both sexual victimization and revictimization remain unclear. Because a viable solution for eliminating men's sexually aggressive behavior remains elusive, research

Elizabeth A. Yeater, Department of Psychology, University of New Mexico; Teresa A. Treat, Department of Psychology, Yale University; Richard J. Viken and Richard M. McFall, Department of Psychology, Indiana University.

We are very grateful to Angela D. Bryan for her advice on an earlier version of this article and to Kathryn L. Lenberg and Jenny K. Rinehart for their assistance with data collection.

Correspondence concerning this article should be addressed to Elizabeth A. Yeater, Department of Psychology, University of New Mexico, Logan Hall, Albuquerque, NM 87131-1161. E-mail: eyeater@unm.edu that investigates factors that place women at risk for sexual victimization is necessary.

One cognitive factor, risk recognition ability, has been the focus of numerous empirical investigations (for a comprehensive review, see Gidycz, McNamara, \& Edwards, 2006). This research posits that women at high risk for sexual victimization (i.e., previously victimized women), relative to women at low risk for victimization (i.e., women who have not been victimized previously), demonstrate difficulties recognizing cues that indicate risk for peerrelated sexual revictimization. As a consequence, they are thought to remain longer in sexually risky situations, thus increasing the probability that they will be sexually victimized.

Empirical support for the risk recognition hypothesis has been mixed, with some studies demonstrating a relationship between deficient risk recognition and a history of sexual victimization (e.g., Soler-Baillo, Marx, \& Sloan, 2005; Wilson, Calhoun, \& Bernat, 1999) and others failing to do so (e.g., Messman-Moore \& Brown, 2006; Yeater, Viken, McFall, \& Wagner, 2006). The reasons for these contradictory findings remain unclear. Different methods and definitions of sexual victimization have been used to test this relationship, however, perhaps contributing to these inconsistent results (Gidycz et al., 2006).

Despite differences, these studies share a focus on comparing the average risk judgments of victimized and nonvictimized 
women. This approach, while informative, does not identify specific cognitive processes that account for these judgments. Indeed, women's risk judgments could arise from multiple processes, including greater or lesser difficulty in detecting cues of risk or a tendency to view situations in general as more or less risky. Within the broader study of perception in cognitive science, it is common to use models that distinguish among multiple explanations for participant responses. The well-known signal detection theory approach (Green \& Swets, 1966) distinguishes between two unobserved processes that can lead to differences in responding: perceptual sensitivity and decisional bias. Perceptual sensitivity is the ability to distinguish levels of risk. Applied to the evaluation of victimization risk, a more sensitive participant will be better able to differentiate and use cues that are associated with risk, so her judgments will correspond more closely to the level of risk present in a situation. In contrast, decisional bias indexes the boundary or threshold at which a participant judges a situation to be risky. Thus, participants with a lower threshold for riskiness will require less evidence of risk before classifying a situation as risky. These participants will view many situations as risky, even those in which only weak cues of risk are present. In contrast, participants with a higher threshold for judging risk will classify few situations as risky, even those in which strong cues of risk are present.

Past findings demonstrating differences in women's risk judgments could be due to differences in sensitivity to risk cues or to differences in the threshold for deciding whether a situation is risky. To date, efforts to understand individual differences in women's judgments of victimization risk have not distinguished these two underlying processes. Determining the extent to which sensitivity and bias processes are related to individual differences in women's risk judgments is important, as it would clarify more precisely why such differences exist, as well as potentially inform the development of individual-specific intervention strategies that presumably would be more effective than existing approaches. For example, women who engage in behaviors that increase their risk of victimization (e.g., drinking alcohol with a man in an isolated location) may underestimate risk relative to their peers. This might suggest that such women rely on a higher threshold when judging risk for victimization, such that fewer situations are perceived to be risky. Alternatively, these women might be less sensitive to riskrelevant information, such that they tend, to a greater degree than their peers, to misidentify risky situations as not risky and vice versa.

Of course, social situations are inherently complex. Thus, examining processing of aspects of these situations other than victimization risk would allow us to distinguish specific deficits for processing of risk-relevant information from global deficits in information processing. One such aspect concerns the potential impact of the woman's behavior in these situations on her social acceptance or popularity (hereinafter called popularity impact). Nurius and Norris (1995) posited that a woman's cognitive appraisals of and responses to risk may be disrupted by competing goals and concerns, such as worries over damaging the relationship with the man or being judged negatively by other people. Using ethnographic observation to identify risk factors for rape, Armstrong, Hamilton, and Sweeney (2006) noted that college women reported social pressure to party and "hook up" with men in order to be accepted and found men's sexual advances in social situations to be the basis for their self-esteem and social status. A focus on social acceptance by men also appears to affect women's responses to a sexual assault. For example, Norris, Nurius, and Dimeff (1996) and Turchik, Probst, Chau, Nigoff, and Gidycz (2007) found that concerns over being rejected by men negatively affected undergraduate women's use of active resistance strategies.

Although a number of studies have investigated the link between women's risk judgments and victimization history, few studies have explored other attitudinal, personality, or personal characteristics that might influence these judgments. One exception is rape myth acceptance (Burt, 1980), defined as beliefs that rape is justified or that women are responsible for sexual assault. Loiselle and Fuqua (2007) found that women higher in rape myth acceptance, relative to women lower in rape myth acceptance, showed longer response latencies when asked to indicate when the man had "gone too far" in an audiotaped date rape vignette. In addition, Mason, Riger, and Foley (2004) demonstrated that women higher in rape myth acceptance blamed the female victim more and were less likely to believe rape had occurred in a date rape vignette than were women lower in rape myth acceptance. Finally, Bohner and Lampridis (2004) found that women higher in rape myth acceptance believed that they were less vulnerable to sexual assault and viewed rape-related information as less personally relevant than women lower in rape myth acceptance. Given these findings, women higher in rape myth acceptance might be expected to view the woman, rather than the situation, as being more important in determining victimization risk. If so, they should be less sensitive to situational risk information and less likely to judge situations to be risky. Because research generally has found little or no relationship between women's rape myth acceptance and their history of sexual victimization (e.g., Muehlenhard \& Linton, 1987), rape myth acceptance represents an individual difference variable that may make an independent and important contribution to the prediction of women's judgments of victimization risk.

\section{The Present Study}

The current study extends previous research on the relationship between undergraduate women's risk recognition and individual differences in both victimization history and rape myth acceptance by decomposing the variability in risk judgments into components reflecting (a) sensitivity to risk-relevant and popularity impact information and (b) the decisional threshold for identification of high-risk situations.

To facilitate the differentiation of these cognitive processes, we used an explicit risk classification task in which participants judged the riskiness (high or low) of a large number of vignettes depicting situations that varied in their degree of sexual victimization risk (i.e., from low risk to high risk) and popularity impact. Experts in the area of sexual violence provided normative ratings of the riskiness of each situation, as such individuals are considered to be particularly aware of the various facets of risk information such as that presented in the vignettes. Participants who are sensitive to this information would be expected to make decisions about the riskiness of the vignettes that map well onto the ratings of experts. Undergraduate women provided normative judgments of the popularity impact of each vignette, because members of the peer group described in the vignettes would be better judges of the 
consequences of the woman's behavior on her popularity than would experts.

We estimated sensitivity to normative risk and popularity impact by regressing individual participants' dichotomous risk judgments for each vignette on the normative risk and popularity impact information for each vignette. The resulting regression slopes index each participant's utilization of normative risk or popularity impact information when making explicit risk judgments. In the text, we use the term utilization when referring to our specific analytic approach and the term sensitivity when referring to the broader theoretical concept of perceptual sensitivity. Conveniently, the intercept estimate in these individual-specific regression analyses also provides an index of the decision boundary, or threshold, which separates high- and low-risk situations for each participant. Overall, therefore, the explicit risk classification task allowed us to estimate for each participant her decisional bias in estimating risk, her sensitivity to the risk-relevant information depicted in the vignettes, and her sensitivity to information that might distract her from recognizing risk (i.e., popularity impact).

In addition to facilitating separate estimates of sensitivity and bias, the configuration of the explicit risk classification task allowed us to address a persistent problem with past research. The majority of these studies have used vignettes that describe only high-risk situations (Gidycz et al., 2006). Given this, it is unclear whether victimized and nonvictimized women differ specifically in their evaluation of high-risk situations or whether their evaluations differ generally across situations that vary more widely in riskiness. Gidycz et al. (2006) called for the use of low-risk situations in sexual risk perception studies to evaluate whether women at risk for sexual victimization evidence similar responses to low- and high-risk situations. In the present study, including situations that varied in their degree of victimization risk allowed us to determine whether participants are sensitive to gradients of victimization risk, which is not possible when only high-risk situations are evaluated.

Like the tasks used in past risk perception research (e.g., Messman-Moore \& Brown, 2006; Wilson et al., 1999), our risk classification task explicitly instructed participants to evaluate the riskiness of situations with regard to their potential for unwanted sexual activity. To understand the cognitive influences on the decisions that people make in explicit tasks, it can be useful to probe their information processing with more implicit tasks that do not explicitly state the intended focus of evaluation (e.g., riskrelated information). The use of implicit tasks also may increase the generalizability of procedures beyond the laboratory in the present context, because women are unlikely to be prompted to attend to victimization risk as directly in real life as they are in explicit risk evaluation tasks (Gidycz et al., 2006). Thus, in the current study, participants completed an implicit attention task prior to the explicit risk classification task. We used a prototype classification task (Treat, Viken, Kruschke, \& McFall, 2010; Viken, Treat, Nosofsky, McFall, \& Palmeri, 2002), in which participants first viewed two prototypical vignettes reflecting important differences in both victimization risk and popularity impact. Participants then classified additional vignettes as more similar to one or the other prototype, without experimenter instruction or feedback about the basis for their decisions. Participants' classifications allowed us to estimate their relative attention to victim- ization risk versus popularity impact when evaluating the vignettes.

We also included an experimental manipulation of participants' processing of either victimization-risk or popularity-impact information. All participants completed a category learning task, in which they read a series of vignettes and classified each of them into one of two undefined categories. Participants received feedback about the accuracy of their classifications, which was based on either the level of victimization risk or the level of popularity impact present in the vignettes. They were not directed to attend to either stimulus dimension, however, unlike in the explicit classification task. Although the dimensions underlying the categories were never named, feedback based on those dimensions allowed participants to learn to classify the vignettes correctly. This task provided us with two additional predictors of sensitivity and bias parameters in the explicit risk classification task: (a) individual differences in the speed with which participants learned taskrelevant aspects of the situations and (b) the focus of recent learning about social situations (i.e., learning victimization risk vs. popularity impact). Manipulation of learning experience (i.e., learn victimization risk vs. learn popularity impact) should affect utilization of risk- and popularity-relevant information such that participants rely more on the information that they learned when making explicit risk judgments. If this proves to be the case, then the learning task might point us in the direction of interventions that could be useful in changing women's understanding and identification of risky situations.

With respect to specific predictions, we expected that (a) more severe victimization history would be associated with higher thresholds for judging the vignettes as risky, lower sensitivity to situational variation in risk as assessed by expert judgments of risk, and higher sensitivity to situational variation in popularity impact as assessed by undergraduate peers; (b) higher rape myth acceptance would also be associated with higher thresholds for judging risk and lower sensitivity to situational variation in expert-judged risk; (c) greater relative attention to either victimization risk or popularity impact in the prototype classification task would predict greater utilization of this information in the explicit risk classification task; (d) experience learning the different category structures would affect utilization of risk and popularity information in the subsequent explicit classification task; and (e) experience learning the risk category structure would reduce the association between utilization of risk in the explicit risk classification task and both victimization history and rape myth acceptance.

\section{Method}

\section{Participants}

Participants were 194 undergraduate women selected from the psychology subject pool at a medium-sized, southwestern university. The sample was $52.1 \%$ freshman, $95.9 \%$ heterosexual, and $87.6 \%$ single. The sample was diverse ethnically, including $46.6 \%$ White, 36.6\% Hispanic/Latino, 8.4\% Native American, 3.7\% African American, 2.6\% Asian, and 2.1\% "other." Mean age was 19.52 years $(S D=1.39)$. Because the vignettes depicted situations that unmarried, heterosexual, or bisexual undergraduate women between the ages of 18 and 24 might face when dating or interacting socially with men, 25 women who were married, lesbian, or 
over the age of 24 years were excluded from all analyses. One additional participant's data were dropped because she did not complete the cognitive tasks.

\section{Self-Report Measures}

Demographics questionnaire. A self-report measure asked participants for their age, marital status, ethnic membership, and academic status.

Sexual Experiences Survey (SES). The SES (Koss et al., 1987) is a 10-item self-report questionnaire developed to measure various degrees of severity of sexual victimization (i.e., unwanted sexual contact, sexual coercion, attempted rape, and rape) since the age of 14 years. The SES uses behaviorally specific definitions of sexual assault and asks participants to indicate whether the event did or did not occur. Koss and Gidycz (1985) reported that the SES had an internal consistency of $\alpha=.74$, a one-week test-retest reliability of $r=.93$, and a correlation of $r=.73$ with interview responses, suggesting that the SES is a reasonable measure of self-reported sexual victimization.

Following the common scoring procedure for the SES, participants were assigned to a severity category based on the most severe victimization experience they reported having since the age of 14 years. Participants' sexual victimization experiences were coded as: $0=$ no unwanted experiences; $1=$ unwanted sexual contact; 2 = sexual coercion; $3=$ attempted rape; and $4=$ rape. This type of scoring procedure has been used in previous studies (e.g., Gidycz et al., 1993) and reflects the dimensional conceptualization of sexual victimization as described by the authors of the SES (Koss et al., 1987). With respect to frequency of sexual victimization, $45.9 \%$ of our participants reported no history of sexual victimization, $13.4 \%$ reported unwanted sexual contact, $12.9 \%$ reported sexual coercion, $10.8 \%$ reported attempted rape, and $17.0 \%$ reported rape.

Rape Myths Acceptance Scale (RMAS). The RMAS (Burt, 1980) is an 11-item self-report questionnaire that measures the extent to which a person believes rape is justifiable and that women are responsible for being sexually assaulted. Burt reported an alpha coefficient of .88 for this scale. Higher RMAS scores have been found to predict greater acceptance of sexual violence against women (Burt, 1980). This scale was chosen, as opposed to other rape myth acceptance measures, because of its continued use with women in the research literature (e.g., Mason et al., 2004) and because of recently published work demonstrating a relationship between women's rape myth acceptance using the RMAS and poorer risk perception (Loiselle \& Fuqua, 2007). Scores were created by summing participants' responses to the items. In the current study, the mean RMAS score was $26.84(S D=9.30)$, and the internal consistency was .76.

\section{Vignettes}

In a series of preliminary studies (Yeater, McFall, \& Viken, in press; Yeater et al., 2006), a set of written vignettes was developed that described a wide range of problem situations that undergraduate women might face when dating or interacting socially with men. In the first step, undergraduate women provided written descriptions of actual situations they face in their dating and social life. In the second step, a new group of undergraduate women evaluated how risky each situation was in terms of the situation ending in forced sexual activity. In the final step, the descriptions and ratings were used to construct 71 unique vignettes that depicted situations that undergraduate women are likely to experience when dating or interacting socially with men.

Variation in the presence of known risk factors also was present across vignettes. Thus, the vignettes described different contexts (e.g., date, party, bar, library, gym) and types of relationships with the man (e.g., boyfriend, acquaintance, stranger). Because women are more likely to be victimized by someone they know, the majority of the vignettes depicted a boyfriend, a date, or an acquaintance. In addition, some of the vignettes described putative risk factors for sexual victimization (e.g., alcohol intoxication, social isolation, dating a man who exhibits characteristics associated with sexual aggression), contextual cues signaling a possible impending assault (e.g., man making verbal threats to obtain sexual activity, touching the woman without her consent, attempting to get the woman intoxicated), or possible threats to women's popularity or social acceptance (e.g., pressure by popular men or peers to engage in potentially risky behaviors). Four experts with multiple publications in the area of sexual violence, and four graduate students working in the labs of those investigators, provided the normative risk ratings of the vignettes. The expert raters evaluated how risky each situation was in terms of the woman having an unwanted sexual experience $(1=$ not risky; $5=$ completely risky). An unwanted sexual experience was defined as one in which the woman is verbally or physically coerced into having sexual contact of any kind with a man. Thirty undergraduate women provided the normative ratings of popularity impact by rating how much the woman's behavior in each situation was likely to influence her popularity $(1=$ none; $5=$ quite a lot $)$. Popularity was defined as how much the woman was valued, liked, or socially accepted.

Intercoder agreement was assessed by intraclass correlation coefficients (ICCs). The ICC across raters and vignettes for the victimization risk dimension was .96 ; for the popularity impact dimension, the ICC was .88. A mean sexual victimization risk rating was assigned to each vignette on the basis of the normative ratings provided by the experts, and a mean popularity impact rating was assigned to each vignette on the basis of the normative ratings provided by the undergraduate women. The mean risk rating for the 71 vignettes was $3.10(S D=1.14)$; the mean popularity impact rating was 2.79 $(S D=0.50)$. The ratings for the two dimensions had a low and nonsignificant correlation $(r=-.12)$, suggesting that the dimensions were relatively independent. None of the participants involved in the vignette development process participated in the current study.

\section{Procedure}

The study was conducted in compliance with the university's Internal Review Board. Participants first provided informed consent and then completed the tasks on a computer in a private room. Same-sex experimenters assisted the participants through the study procedures. To ensure that the instructions for the explicit classification task did not influence participants' approach to the more implicit tasks, we presented the prototype classification tasks first, followed by the category learning and explicit classification tasks. After finishing the tasks, participants completed the demographics 
questionnaire, the SES, and the RMAS. The study took approximately $1 \mathrm{hr}$ to complete.

\section{Prototype Classification Tasks}

The prototype classification tasks estimated participants' relative attention to the victimization risk and popularity impact information in the vignettes. In these tasks, participants classified the vignettes as being more like one of two prototypes, without explicit instructions about the basis on which they should make their classifications. From the normative ratings provided by the experts and undergraduate women, two pairs of prototypes were chosen. Each pair varied substantially along both the victimization risk and popularity impact dimensions so that participants could rely on either or both dimensions when making their classifications. For example, one pair of prototypes included a vignette characterized by low sexual victimization risk and low popularity impact, as well as a vignette characterized by high victimization risk and high popularity impact:

A low sexual victimization risk, low popularity impact vignette: A woman is at home on a Thursday evening. She checks her e-mail and finds a message from a guy in her sociology class. He asks her whether she wants to meet him at the library on Sunday to study for an upcoming test.

A high sexual victimization risk, high popularity impact vignette: A woman has been dating a guy for about a month. She really likes him. He's very attractive and lots of women on campus want to date him. She's felt kind of special that he's chosen her rather than someone else. One night, she's making out with him and things start to get pretty hot. She doesn't want to have sex with him yet, but she can tell he's really into it. He tells her that if she doesn't have sex with him, he'll find someone else who will.

Participants classified 24 vignettes in each of two tasks, six from each quadrant defined by the sexual victimization risk (high or low) and popularity impact (high or low) dimensions. In one of the prototype classification tasks, participants read the low-risk/highpopularity prototype and the high-risk/low-popularity prototype. Participants then viewed 24 vignettes and classified each as more like one of the two prototypes. The degree to which a participant classified, for instance, high-risk/high-popularity impact vignettes with Prototype 1 (because the vignettes matched on popularity) as opposed to Prototype 2 (because they matched on risk) provided information about the participant's relative attention to popularity impact in comparison with attention to victimization risk when evaluating the vignettes. In the other prototype classification task, participants read the high-risk/high-popularity and the low-risk/ low-popularity prototypes, viewed 24 new vignettes, and again classified each of them with one of the two prototypes. Participants completed both prototype classification tasks, but their order was counterbalanced across participants.

Category learning task. The category learning task manipulated recent learning about social situations (i.e., learning a category structure based on victimization risk vs. popularity impact). In this task, participants classified 16 vignettes into one of two categories, which were labeled arbitrarily as Categories V and N. Feedback to participants was based on the norms for either victimization risk (with 8 high- and 8 low-risk situations) or popularity impact (with 8 high- and 8 low-popularity situations). Par- ticipants were assigned randomly to experience one of the two category structures (i.e., sexual victimization risk or popularity impact). Participants viewed each of the vignettes, indicated the category to which it belonged, and received feedback about whether their classifications were correct or incorrect. Participants were not told the basis for the feedback. Participants completed four blocks of the category learning task; the same 16 vignettes were presented in each block.

Explicit classification task. The explicit classification task estimated participants' sensitivity and bias to victimization risk and popularity impact information when making explicit risk judgments. All 71 vignettes were used in this task, including those vignettes used in the prototype classification and learning tasks. Participants were given the following instructions:

Please read each of the following vignettes. After reading each vignette, you will be asked to evaluate how risky the situation is in terms of the woman having an unwanted sexual experience. By unwanted, we mean a sexual experience she will feel bad about, be hurt by, or regret later. You will classify each situation as either low risk or high risk.

A broad definition of unwanted sex was used instead of one that included words such as sexual coercion or assault, as research shows that women often adhere to stereotypes of rape (i.e., stranger rape) when considering risk for victimization (e.g., Hickman \& Muehlenhard, 1997). Moreover, these words were excluded to avoid participant priming (Wilson et al., 1999).

\section{Analytic Strategy}

Participant-specific indices of performance on both the prototype classification and category learning tasks first were derived for inclusion as predictors in analyses of the explicit risk classification task.

Prototype classification tasks. Hierarchical linear modeling (HLM 6.0) was used to fit a multilevel, logistic regression model to participants' implicit classification judgments. For each of the two tasks, the centered victimization risk and popularity impact normative ratings for each vignette were included as two predictors of participants' dichotomous classifications of the 24 vignettes. The estimated beta weights for these two predictors indicated each participant's attention to victimization risk and popularity impact information when making classification judgments. For example, a coefficient of .64 for victimization risk would indicate that the log-odds of classifying a presented stimulus with the high-risk prototype increased by .64 for every $1-S D$ increase in the risk normative ratings. In prototype classification tasks such as the one we used, attention to the two dimensions necessarily will show a strong negative correlation. Thus, we computed an index of relative attention to victimization risk and popularity impact from the empirical-Bayes estimates for each of the two classification tasks. Within each task, we computed the difference between the absolute values of the victimization risk and popularity impact coefficients. These difference scores were averaged across the two tasks, and the resulting relative attention index was centered for inclusion as a predictor in analyses of the explicit classification task. The relative attention index was scaled so that more positive values indicated relatively greater attention to victimization risk than to popularity impact, whereas more nega- 
tive values indicated relatively greater attention to popularity impact than to victimization risk. Thus, this procedure provided for each participant an index of her relative attention to victimization risk compared with popularity impact information in an implicit categorization task in which she was free to use any aspect of the vignettes in making her classifications.

Category learning task. HLM 6.0 was used to fit a multilevel, linear regression model to participants' percentage-correct scores for each of the four blocks of the category learning task. To facilitate interpretation of parameter estimates, we recoded values for the four blocks (1-4) as 0 to 3 . We used the growth curve approach to modeling change in performance associated with learning. The intercept and slope estimates indexed average percentage correct on the first block and average increase in percentage correct on each successive block, respectively. Centered empirical-Bayes estimates of each participant's intercept and slope for the category learning task were included as predictors in analyses of the explicit risk classification task.

Explicit risk classification task. HLM 6.0 was used to fit a two-level, logistic regression model to participants' explicit classifications of 71 vignettes as high or low risk. Reported statistics are for a unit-specific model with robust standard errors. In the Level 1 equation, centered victimization risk and popularity impact normative ratings for each vignette were included as predictors of participants' explicit classifications. In other words, within each participant, the 71 dichotomous classification judgments were regressed onto the average normative data for each of the 71 vignettes. The beta weights at Level 1 estimated each participant's utilization of victimization risk and popularity impact information (i.e., their utilization scores) when making explicit classifications. The intercept at Level 1 indicated for each participant the location of the decision boundary (threshold) separating high- and low-risk situations. Thus, the Level 1 equation specified the link between the normative data and each participant's classifications, and it contained three parameters: (a) the log-odds of making high-risk classifications (i.e., the location of the decision boundary); (b) the utilization of victimization risk information when making these classifications; and (c) the utilization of popularity impact information when making these classifications.

These three parameters then become dependent variables in the three Level 2 equations, which specify multiple predictors of these three characteristics of performance on the explicit classification task (i.e., there is one equation per parameter). Six main effect predictors of variation in these three parameters were examined: (a) relative attention to victimization risk versus popularity impact (centered) in the prototype classification task, which becomes more positive as attention to victimization risk increases, relative to attention to popularity impact; (b) learning intercept (centered) in the category learning task, which indicated performance on the first block; (c) learning slope (centered) in the category learning task, which indicated average change in performance on each block; (d) category structure experienced in the category learning task $(-1=$ victimization risk structure; $1=$ popularity impact structure), which was assigned randomly as part of the experimental design and was orthogonal to the other predictors; (e) victimization history (centered); and (f) rape myth acceptance (centered). We also included the two-way interactions between either victimization history or rape myth acceptance and either category structure, learning intercepts, or learning slopes. Three-way interactions in- volving victimization history or rape myth acceptance, category structure, and either learning intercepts or learning slopes were included as well. Overall, therefore, the model evaluates multiple predictors of each of the three indices of performance on the explicit classification task. Only significant main effects and the highest order significant interactions involving either victimization history or rape myth acceptance are reported below.

\section{Results}

\section{Average Threshold and Utilization Estimates in Level 1 Equation}

The average log-odds of a high-risk classification (i.e., the threshold) was .80 , indicating that $69 \%$ of the vignettes, on average, were classified as high risk, $t(177)=13.77, p<.001, d=$ 2.07 (see Oishi, Lun, \& Sherman, 2007, for further information on computation of effect sizes in multilevel models). Significant positive coefficients for victimization risk and popularity impact indicated that the probability of making a high-risk classification increased as victimization risk increased, $b=1.98, t(177)=$ 38.28, $p<.001, d=5.75$, and as popularity impact increased, $b=$ $0.30, t(177)=4.05, p<.001, d=0.61$. The markedly stronger link between victimization risk and participants' explicit risk classifications is consistent with the explicit instructions to participants to classify vignettes on the basis of risk. Nonetheless, as the potential popularity impact of a woman's decision increased, perception of risk increased.

\section{Predictors of Threshold Location in Level 2 Equation}

Variability in participants' thresholds for classifying situations as high risk was associated with two predictors. First, victimization history negatively predicted the probability of making a high-risk classification, $b=-0.16, t(177)=-2.87, p<.01, d=-0.43$, as expected. As severity of victimization history increased, fewer vignettes were classified as high risk. To illustrate the effect, we note that the model-predicted percentage of situations classified as high risk by women reporting the most severe victimization experience was 59.82, whereas the predicted percentage of high-risk classifications by women reporting no victimization history was 73.62. Because we estimated a linear model, the model-predicted percentages of high-risk classifications for women reporting victimization experiences between these two extremes were intermediate. The top panel of Figure 1 displays this victimization-linked difference in the threshold for high-risk situations. The centered normative ratings for victimization risk are displayed along the $x$-axis, and the probability of a high-risk classification is presented on the $y$-axis. The logistic function linking these two variables is displayed for participants with the least and most severe victimization histories. The threshold demarcates the probability of a high-risk classification when risk is at an average value (i.e., at a value of zero, because the normative ratings are centered).

Second, the interaction between rape myth acceptance and category structure was significant, $b=-0.02, t(177)=-2.23, p<$ $.05, d=0.34$. Follow-up characterizations of simple slopes indicated that among participants who experienced the popularity category structure, rape myth acceptance was associated with the threshold, such that women who endorsed more rape myths clas- 

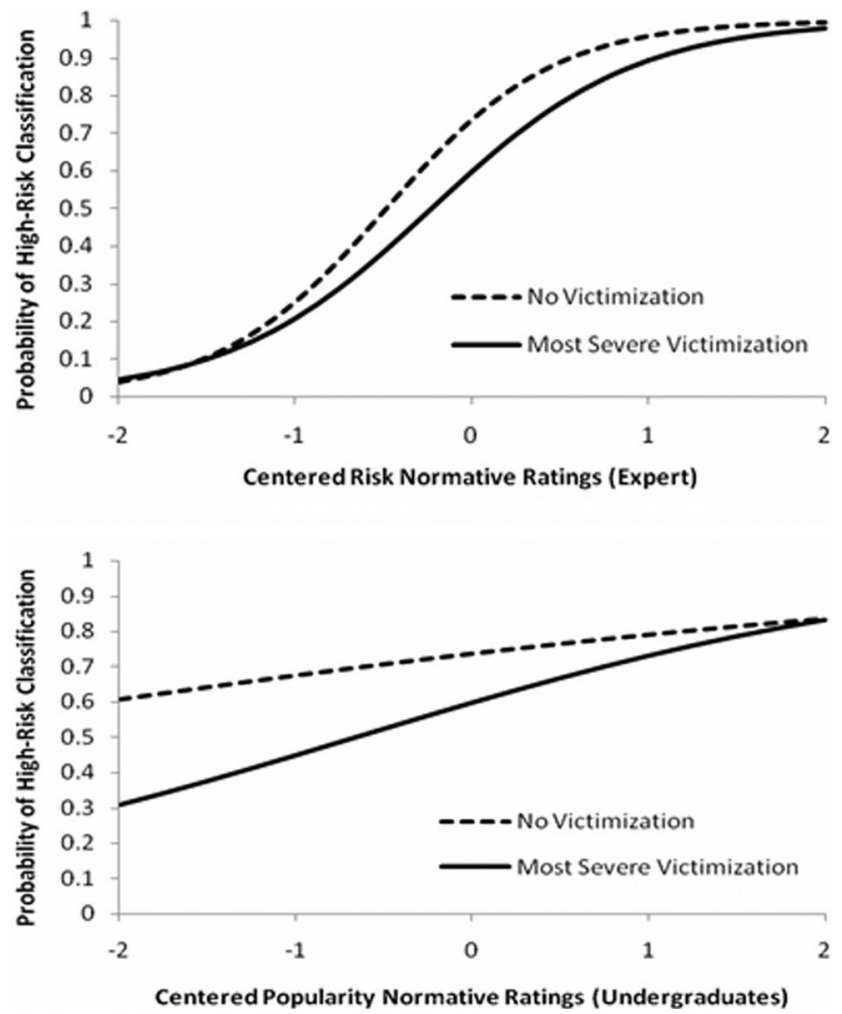

Figure 1. Main effect of victimization status on threshold, victimization risk utilization, and popularity impact utilization parameters in the explicit classification task. For ease of interpretation, only the two most extreme levels of victimization status are presented (no victimization and most severe victimization). The upper panel documents that those reporting more severe victimization histories classified fewer situations as high risk and relied to a lesser degree on normative victimization risk information. The lower panel indicates that those reporting more severe victimization histories relied to a greater degree on popularity impact information when making explicit risk judgments.

sified fewer vignettes as high risk, $b=-0.03, t(88)=-2.91, p<$ $.01, d=-0.67$. This implies that participants whose rape myth acceptance was $1 S D$ above the mean classified $61.86 \%$ of the vignettes as high risk, whereas those with scores $1 S D$ below the mean classified $72.05 \%$ of the situations as high risk. In contrast, variation in the threshold was unrelated to rape myth acceptance among participants who experienced the risk category structure, $b=0.01, t(86)=0.80, n s, d=0.17$.

\section{Predictors of Victimization Risk Utilization in Level 2 Equation}

Variability in participants' reliance on victimization risk information when making explicit judgments about risk was associated with numerous predictors. First, relative attention to victimization risk versus popularity impact on the prototype classification tasks influenced the extent to which participants relied on risk when making their explicit risk classifications, $b=0.19, t(177)=2.28$, $p<.05, d=0.34$. As predicted, women who attended relatively more to victimization risk than to popularity impact relied to a significantly greater degree on the victimization risk information when classifying the vignettes as high or low risk.

Second, category structure influenced victimization risk utilization. Consistent with our expectations, participants who experienced the risk category structure relied significantly more on victimization risk when making their explicit risk classifications, $b=-0.22, t(177)=-3.65, p<.001, d=-0.55$. Thus, exposure to the risk category learning experience presumably sensitized participants to the victimization risk information in the vignettes. Participants who showed better performance on the first block of either learning task also showed greater use of victimization risk on the explicit classification task, $b=0.01, t(177)=2.38, p<.05$, $d=0.36$.

Third, victimization history negatively predicted reliance on risk information, $b=-0.09, t(177)=-2.34, p<.05, d=-0.35$. On average, participants who reported the most severe victimization history showed an average victimization risk utilization score of 1.74, whereas participants without a victimization history showed an average victimization risk utilization score of 2.11. This victimization-linked difference in utilization of victimization risk information is displayed in the top panel of Figure 1. The decreased reliance on victimization risk information by participants reporting the most severe victimization histories is indicated by the shallower slope of the function linking normative risk information with high-risk classification probabilities. In contrast, the slope of the logistic function for those with no victimization history is steeper, indicating greater utilization of victimization risk information when making explicit judgments about risk.

The predicted three-way interaction between victimization history, category structure, and learning intercepts, $b=-0.01, t(177)=$ $-2.04, p<.05, d=-0.31$, was decomposed by examining the two-way interactions between victimization history and learning intercepts separately for participants who had experienced the risk and popularity category structures. The two-way interaction between victimization history and learning intercepts was significant among participants who experienced the risk category structure, $b=0.02, t(86)=2.91, p<.01, d=0.63$, but not among participants who experienced the popularity category structure, $b=0.00, t(88)=0.08, n s, d=0.02$. Further decomposition of the former two-way interaction indicated that participants whose performance on the first block of the risk category structure was above the median showed no relationship between victimization history and victimization risk utilization when making explicit risk classifications, $r(49)=-.071, n s$. In contrast, participants who showed below median performance when exposed to the risk category structure showed a significant negative association between victimization history and victimization risk utilization when making explicit risk classifications, $r(47)=-.369, p<.05$. This two-way interaction of victimization history and learning intercepts on victimization risk utilization for those experiencing the risk category structure is displayed in Figure 2. Those who reported the most severe victimization history and performed below the median on the first block of the risk category structure showed significantly lower utilization of victimization risk information when judging risk explicitly.

Fourth, rape myth acceptance negatively predicted reliance on risk information, $b=-0.02, t(182)=-3.22, p<.01, d=-0.48$, as expected. Participants whose rape myth acceptance was $1 S D$ above the mean showed an average victimization risk utilization 


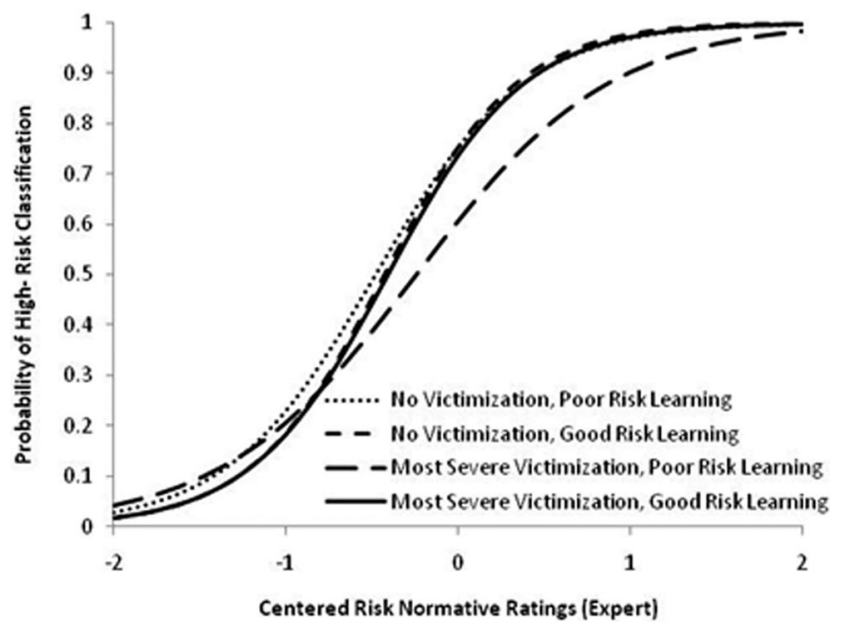

Figure 2. Interactive effect of victimization status and learning intercepts for those experiencing risk category structure on utilization of victimization risk in the explicit classification task. Those reporting the most severe victimization history who performed above the median on the first block of the risk category structure ("good risk learning") showed greater utilization of victimization risk information when making explicit risk judgments relative to those reporting the most severe victimization history who performed below the median on the first block of the risk category structure ("poor risk learning"). Victimization risk utilization was unrelated to performance on the risk category structure among those reporting no victimization history.

score of 1.74; participants with scores $1 S D$ below the mean showed an average utilization score of 2.19. Figure 3 presents this finding. The shallower slope of participants endorsing more rape myths indicates their decreased utilization of victimization risk information when making explicit judgments about risk.

The predicted three-way interactions between rape myth acceptance, category structure, and either learning intercepts or learning slopes were significant, $b \mathrm{~s}=-0.00,-0.01, t \mathrm{~s}(177)=-3.59$, $-3.12, p \mathrm{~s}<.01, d \mathrm{~s}=-0.54,-0.47$. Significant two-way interactions emerged between rape myth acceptance and learning intercepts or learning slopes only among participants who experienced the risk category structure, $b \mathrm{~s}=0.00,0.02, t \mathrm{~s}(986)=3.15$, $2.68, p \mathrm{~s}<.05, d \mathrm{~s}=0.68,0.58$. Further decomposition of these two-way interactions indicated that (a) participants whose performance on the first block of the risk category structure was above the median showed a nonsignificant relationship between rape myth acceptance and risk utilization when making explicit risk classifications, $r(47)=-.250, n s$, whereas participants who performed below the median on the first block showed a significant negative association between rape myth acceptance and risk utilization when judging risk explicitly, $r(49)=-.392, p<.01$, and (b) participants whose learning rate on the risk category structure was above the median showed a nonsignificant relationship between rape myth acceptance and risk utilization when making explicit classifications, $r(48)=-.181, n s$, whereas those whose learning rate was below the median showed a significant negative relationship between rape myth acceptance and risk utilization when making explicit risk classifications, $r(48)=-.405, p<.01$. Note that this pattern of findings is the same as that observed for the interaction between victimization history and learning inter- cepts (for those completing the risk category structure) and depicted in Figure 2.

\section{Predictors of Popularity Impact Utilization in Level 2 Equation}

Participants' utilization of popularity information when judging risk explicitly also was associated with three significant predictors. First, category structure influenced popularity utilization, such that participants who experienced the popularity impact structure relied significantly more on popularity information, $b=0.28, t(177)=$ $3.10, p<.01, d=0.47$, when making explicit risk classifications, consistent with expectations. Specifically, participants who experienced the popularity impact structure showed an average popularity impact utilization score of 0.58 . Those participants who experienced the risk structure, in contrast, showed an average utilization score of 0.01 . Second, just as was true for victimization risk utilization, participants who showed better performance on the first block of either learning task also showed greater use of popularity information in the explicit risk classification task, $b=$ $0.01, t(177)=2.12, p<.05, d=0.32$

Third, as expected, victimization history positively predicted popularity impact utilization, $b=0.12, t(177)=2.14, p<.05$, $d=0.32$; as severity of victimization history increased, utilization of popularity impact information when judging risk also increased Participants who reported the most severe victimization history showed an average popularity impact utilization score of .60, whereas participants without a victimization history showed an average score of .12. The lower panel of Figure 1 displays this effect. In this case, the $x$-axis presents centered normative ratings for popularity impact, rather than victimization risk. The function linking these normative data to the probability of a high-risk classification remains logistic, but the nonlinear components of the function largely lie outside the range of the normative ratings (which, centered, range from -2 to +2 ). The far shallower slope of the logistic function in the lower panel of Figure 1, relative to that of the upper panel of Figure 1, indicates that variation in the

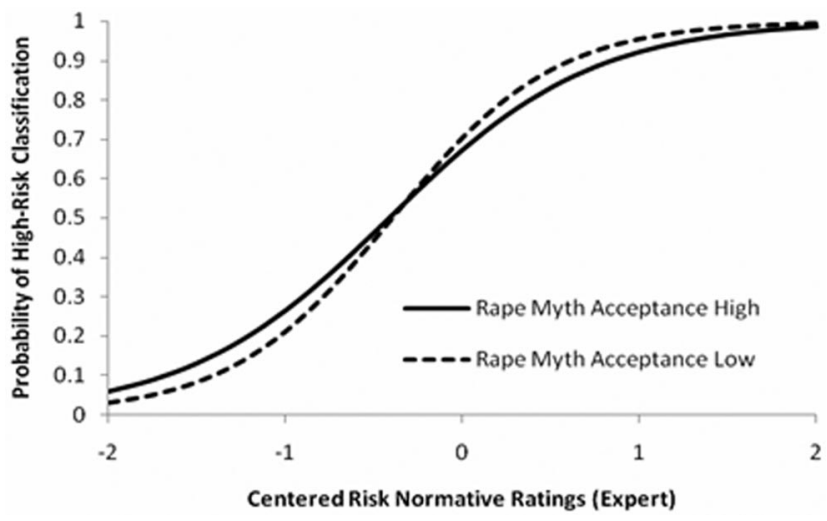

Figure 3. Main effect of rape myth acceptance on victimization risk utilization in the explicit classification task. For ease of interpretation, only two levels of rape myth acceptance are displayed (1 SD above the mean and $1 S D$ below the mean). Those endorsing more rape myths relied less on normative victimization risk information when making explicit risk judgments. 
popularity impact of situations had a much smaller impact on explicit risk judgments than did variation in the victimization risk of situations, as would be expected. Nonetheless, the nonzero slope linking the popularity impact norms to the probability of a highrisk classification indicates that popularity impact did exert an influence on explicit risk classifications. The steeper slope for those reporting the most severe victimization history indicates that these participants relied on popularity impact information to a significantly greater degree than participants reporting no victimization history when making explicit judgments about risk.

\section{Ethnicity as a Predictor}

The extent to which ethnicity moderates the effects outlined above is an important topic for future research with larger samples than the one in the present study. Although power was insufficient to include ethnicity as a moderator in our HLM analyses, we conducted exploratory analyses of the explicit classification data provided by the two largest ethnic subgroups in our sample (Hispanic and White). The analyses revealed that ethnicity showed neither statistically nor practically significant associations with the probability of making a high-risk classification or with the utilization of either victimization risk or popularity impact when making explicit risk judgments.

\section{Discussion}

A major focus of the present work was to examine whether sensitivity to and bias for risk-relevant information are related to young, undergraduate women's victimization history and endorsement of rape myths. As expected, women with more severe victimization histories, relative to women with less severe victimization histories, perceived fewer situations to be high risk and relied less on victimization risk information when judging risk explicitly. These results are consistent with past research demonstrating a relationship between poorer risk recognition and a history of sexual victimization (e.g., Soler-Baillo et al., 2005; Wilson et al., 1999). However, the present study is the first in this area to distinguish sensitivity and bias processes in risk recognition, thereby extending past research by providing a more thorough understanding of the cognitive processes that may underlie victimization-linked variability in women's judgments of victimization risk.

The implications of our findings for previously victimized women are troubling. Given that women with more severe victimization histories require more evidence of risk to conclude that a situation is unsafe (i.e., they have a higher threshold for perception of high-risk situations), they may be inclined to conclude that situations are less risky than they are in reality. This might be associated with entering or remaining in situations having a high potential for victimization. Additionally, women with more severe victimization histories who are less sensitive to risk may have difficulty discriminating and using contextual cues that signal this risk. This too might be linked to decisions to approach or stay in potentially dangerous situations.

Consistent with hypotheses, women with more severe victimization histories also relied on popularity impact information to a greater extent than women with less severe victimization histories when judging risk explicitly. To the best of our knowledge, this is the first study to evaluate whether a theoretically relevant dimension other than victimization risk also influences women's risk judgments. The positive relationship between victimization status and utilization of popularity impact information, in concert with the negative relationship between victimization status and utilization of victimization risk information, highlights the specificity of the deficient processing of risk-relevant information among women with more severe histories of victimization (i.e., global deficits in information processing among victimized women cannot account for the pattern of results). Our results support the view of researchers who have posited that women may experience difficulty recognizing risk because other aspects of potentially dangerous situations, such as fear of losing the relationship with the man, obscure their ability to do so (Norris et al., 1996; Nurius \& Norris, 1995; Turchik et al., 2007). This increased reliance on popularity-relevant information may be associated with less effective responding to risky situations among women with more severe victimization histories. Future work could evaluate this possibility by investigating whether sensitivity to popularity information when judging risk is linked to the selection of less effective responses to high-risk situations.

The observed associations between victimization history and information processing may indicate that sensitivity and bias differences are cognitive risk factors for victimization, or they may reflect an effect of past victimization on cognitive processing of new information. Future longitudinal research profitably could examine whether the observed associations between the two exist prior to, concomitant with, or subsequent to the initial victimization experience. Such prospective studies also would be useful in establishing the degree to which processing of risk-relevant information in the laboratory predicts future behavior and experiences outside the lab. Recent research has shown that women's selfreported strategies for responding to an impending sexual assault predicted their actual use of these strategies later in a real-life situation (e.g., Turchik et al., 2007), supporting the potential utility of laboratory-based assessments for our understanding and prediction of women's future behavior in the social environment.

Rape myth acceptance also predicted aspects of risk-relevant processing in the current study. As expected, women higher in rape myth acceptance relied significantly less on victimization risk information than did women lower in rape myth acceptance when making their explicit risk judgments. Related research suggests that men higher in rape myth acceptance, relative to men lower in rape myth acceptance, are less sensitive to women's affective cues (Farris, Viken, Treat, \& McFall, 2006). Moreover, such men have particular difficulty perceiving affective cues that signal rejection when women are depicted as provocatively dressed. Thus, endorsement of rape-supportive attitudes appears to interfere with both women's and men's use of information that may help guide effective decision making in heterosexual interactions. Contrary to our prediction, women higher in rape myth acceptance, relative to women lower in rape myth acceptance, did not demonstrate a higher threshold overall for deciding whether a situation was risky when judging risk explicitly. Notably, however, the expected link between rape myth acceptance and threshold location did emerge for those participants who experienced the popularity category structure (but not the risk category structure). In other words, after exposure to the popularity category structure, women higher in 
rape myth acceptance judged fewer situations to be risky in the explicit risk classification task.

Unlike previous research on women's risk perception, we assessed the effects of both victimization history and a theoretically relevant attitudinal variable (i.e., rape myth acceptance) on women's risk judgments. Our results demonstrate the value of expanding the focus of risk-processing research beyond that of adult victimization status alone and suggest several interesting avenues for future work. For instance, it may be informative to explore whether other characteristics, such as childhood sexual abuse (CSA), influence perceptual sensitivity or decisional bias (or both). Messman-Moore and Brown (2006) found that, when asked to imagine themselves in an acquaintance date rape vignette, women who had experienced CSA indicated that they felt uncomfortable earlier than nonvictimized women and women reporting other types of victimization experiences (e.g., adult sexual assault). Thus, we might expect women who have experienced CSA, relative to women reporting adult sexual assault, to have a lower threshold when judging risk. It also could be useful to assess whether the correlates of victimization, such as depression, posttraumatic stress disorder, alcohol abuse, and a history of child or adult physical violence are linked to victimized women's risk judgments.

Consistent with hypotheses, the findings from the implicit attention task suggested that women who were spontaneously attending less to normative aspects of victimization risk were less likely to rely on this information when making risk judgments in an explicit task. By comparison, women who were already attending to victimization risk, even when they were not required to do so, appeared better able to use situation-specific information about risk when making explicit risk judgments. This suggests that some women monitor or attend to information about victimization risk even when it is not required in the moment. Thus, they may be better prepared to use this information when required to do so.

Recent learning about risk and popularity information also influenced women's risk-relevant processing. As expected, participants who were exposed to the risk category structure relied more on risk information when making explicit risk judgments; those who experienced the popularity category structure relied more on popularity information when making the same judgments. Thus, participants relied to a greater degree on information that was congruent with their recent learning history when judging risk explicitly.

In an important interaction, the negative relationship between victimization history and sensitivity to victimization risk information in the explicit classification task was moderated by a combination of category structure and learning performance. In particular, women with more severe victimization histories who showed better first-block performance on the risk category structure used risk information to the same degree as the less victimized women. In contrast, women with more severe victimization histories who showed poorer first-block performance on the risk category structure relied significantly less on risk information. No such patterns emerged for those who were exposed to the popularity category structure. These findings suggest that active learning (i.e., learning with feedback) about risk-relevant information may be a useful component of prevention programs. In fact, prevention researchers are beginning to incorporate active learning into their programs. In one such program, Yeater and O'Donohue (2002) found that women who demonstrated difficulty identifying sexual assault risk factors depicted in vignettes became better at recognizing these cues on successive trials when provided with feedback about the accuracy of their responses. Future research should examine whether more extensive efforts to sensitize participants to riskrelevant information in their social environment (whether experimentally or naturalistically) would serve to decrease the likelihood of future victimization experiences.

Notably, a parallel finding emerged for rape myth acceptance, such that the combination of category structure and learning performance moderated the relationship between rape myth acceptance and sensitivity to victimization risk information in the explicit classification task. In particular, women higher in rape myth acceptance who showed better first-block performance on the risk category structure used risk information to a similar degree as women lower in rape myth acceptance. Women higher in rape myth acceptance who showed poorer first-block performance on the risk category structure, however, used risk information significantly less relative to women lower in rape myth acceptance. These findings highlight the similarity in the way that victimization history and rape myth acceptance are operating with respect to women's processing of risk information, and they suggest that structured efforts to sensitize women more accepting of rape myths to the risk-relevant information in their social environments may prove beneficial.

Given the significant impact of recent learning experiences on risk-relevant processing, future research should endeavor to identify the crucial aspects of the procedures. For instance, a notraining control condition, a sham condition providing exposure to the vignettes but no feedback, and a condition providing random feedback that is unrelated to the content of the vignettes each would be useful in isolating the mechanisms by which training has an impact on thresholds and on the use of risk and popularity information in making risk judgments. In addition, basic research in cognitive science has revealed several distinct processes that can influence category learning (Treat, Kruschke, Viken, \& McFall, in press), including the relative perceived salience prior to training of the underlying dimensions (e.g., victimization risk versus popularity impact), the rate at which participants' attention shifts toward relevant stimulus dimensions and away from irrelevant stimulus dimensions, and the rate at which participants form associations between individual stimuli and the correct category labels. This kind of process-model approach has proven useful in explaining mechanisms of change in men's perceptions of women (Treat, McFall, Viken, \& Kruschke, 2001), as well as in women's perceptions of women (Treat et al., in press), and it may facilitate the development of novel intervention strategies designed to enhance the accuracy of women's risk judgments.

Our findings suggest several additional avenues for future work investigating women's processing of and responses to victimization risk. For example, the procedures used to develop the vignettes in the current study were designed to ensure that the vignettes were representative of the problem situations that undergraduate women face when dating or interacting socially with men. Like those problem situations, the vignettes are heterogeneous and complex, containing multiple contextual cues of risk. In the current study, we used the judgments of expert raters as a way of integrating the risk information present in these multiple cues, and the results show that these expert risk ratings can be used to uncover 
processing differences associated with victimization history and rape myth acceptance. In future work, it would be useful to analyze these associations further by investigating the specific elements of the vignettes (e.g. alcohol use, sexual activity) that influence risk judgments. This kind of component analysis of contextual cues might help to explain the observed differences in risk judgments associated with victimization history by exploring individual differences in the contextual determinants of those judgments.

It also may be informative to investigate whether different definitions of victimization risk influence women's thresholds for and sensitivity to risk-relevant information. In the current study, we used a relatively broad definition of risk. In future work, researchers might consider using definitions that indicate more explicitly a lack of consent on the woman's part. For instance, women could be asked to evaluate how risky the situation is in terms of the woman being verbally or physically coerced into having sexual contact of any kind with the man.

In the present study, we asked women to imagine an anonymous woman rather than themselves in the vignettes. Whereas this is not uncommon in the victimization risk perception literature (e.g., Wilson et al., 1999; Yeater et al., 2006), related research suggests that women asked to compare their own sexual assault risk with that of an anonymous woman estimate their risk to be lower (e.g., Cue, George, \& Norris, 1996; Norris et al., 1996; Yeater, Viken, Hoyt, \& Dolan, 2009), a phenomenon known as "optimistic bias" (Taylor \& Brown, 1988). To date, the mechanisms responsible for this bias are poorly understood. The approach used in the current study offers promise for identifying the cognitive processes that may underlie this phenomenon. For instance, it would be relatively easy to compare women's processing of risk-related information when imagining themselves in the vignettes compared with imagining an anonymous woman. Such a comparison would allow us to establish whether the increase in judged risk for an anonymous woman is attributable to an altered threshold or changed sensitivity.

This study focused on the early stages of perception of and decision making about victimization risk. When confronted with a situation that may lead to a sexual assault, women must also choose an effective response to reduce their risk and then successfully execute that response. Given research linking risk appraisal to participation in high-risk behaviors (e.g., McCoy et al., 1992), the relationship between women's risk judgments and their response choices to risky situations is an important area for future work. For example, in a study of response choices to vignettes similar to those included in the current study, Yeater and Viken (in press) found that victimized women selected more socially acquiescent responses to risky situations than did nonvictimized women. In future research, simultaneous assessment of risk perception and response choice could provide a way to begin delineating the links between early perceptual processing and women's behavior in risky situations.

To date, prevention programs have been relatively ineffective in decreasing women's self-reported rates of sexual victimization (for a recent meta-analysis, see Anderson \& Whiston, 2005). The results of the current study point in the direction of possible interventions that may help alter women's understanding and identification of risky situations. To lower women's decisional threshold for judging risk, the provision of information that helps set the overall level of women's risk for victimization may be important. One approach to changing women's thresholds might be to tailor prevention information to make it personally relevant (Gidycz et al., 2001). For example, women could be provided with individualized feedback about their risk by assessing their current dating and social behaviors. Women's thresholds also could be shaped by feedback provided in a variant of the learning task used in the current study, in which vignettes varied more continuously along the victimization risk dimension.

With respect to enhancing women's sensitivity to risk information, a category learning task would be one approach for improving women's sensitization to this information. This task might also decrease women's sensitivity to popularity impact information when estimating their risk. Notably, some victimized women performed quite poorly on the risk learning task, perhaps placing them at greater risk of future victimization and necessitating the use of specialized training to improve their sensitization to risk information. Such training might involve additional learning trials or the provision of feedback that specifically directs women's attention to the risk cues depicted in the vignettes. Additional avenues to explore in the future development of intervention strategies based on category learning include lengthening training to enhance the duration of learning effects, diversifying the presented stimuli and the conditions of learning to enhance the generalization of learning effects, identifying the processes underlying learning that facilitate increased reliance on risk-relevant information, and making the information personally relevant by asking women to imagine themselves in the vignettes.

In summary, the results of the current study emphasize the importance of distinguishing the role of sensitivity and bias processes in women's explicit judgments of victimization risk. We document multiple influences on explicit risk evaluation, including utilization of victimization risk and popularity impact information, threshold location, implicit attentional processes, speed of learning, recent learning experience, victimization history, and riskrelevant attitudes (i.e., rape myth acceptance). In 1995, Nurius and Norris called for research providing a more thorough understanding of the multilevel influences on women's cognitive appraisal of risk. Our results provide a significant step in that direction by illustrating that both individual differences (i.e., victimization history and rape myth acceptance) and contextual factors (i.e., victimization risk and popularity impact information) influence women's cognitive processing of risk-relevant information. It is our hope that additional research that differentiates the cognitive processes underlying women's risk judgments will inform the development of interventions to assist women in making more accurate judgments of their risk for sexual victimization.

\section{References}

Anderson, L. A., \& Whiston, S. C. (2005). Sexual assault education programs: A meta-analytic examination of their effectiveness. Psychology of Women Quarterly, 29, 374-388.

Armstrong, E. A., Hamilton, L., \& Sweeney, B. (2006). Sexual assault on campus: A multilevel, integrative approach to party rape. Social Problems, 53, 483-499.

Bohner, G., \& Lampridis, E. (2004). Expecting to meet a rape victim affects women's self-esteem: The moderating role of rape myth acceptance. Group Processes and Intergroup Relations, 7, 77-87.

Burt, M. R. (1980). Cultural myths and supports for rape. Journal of Personality and Social Psychology, 38, 217-230. 
Cue, K. L., George, W. H., \& Norris, J. (1996). Women's appraisals of sexual-assault risk in dating situations. Psychology of Women Quarterly, 20, 487-504.

Farris, C., Viken, R. J., Treat, T. A., \& McFall, R. M. (2006). Heterosexual perceptual organization: Application of the choice model to sexual coercion. Psychological Science, 17, 869-986.

Fisher, B. S., Cullen, F. T., \& Turner, M. G. (2000, December). The sexual victimization of college women. Washington, DC: National Institute of Justice and Bureau of Justice Statistics, U.S. Department of Justice.

Gidycz, C. A., Coble, C., Latham, L., \& Layman, M. (1993). Sexual assault experience in adulthood and prior victimization experiences. Psychology of Women Quarterly, 17, 151-168.

Gidycz, C. A., Lynn, S. J., Rich, C. L., Marioni, N. L., Loh, C., Blackwell, L. M., Stafford, J., Fite, R., \& Pashdag, J. (2001). The evaluation of a sexual assault risk reduction program: A multisite investigation. Journal of Consulting and Clinical Psychology, 69, 1073-1078.

Gidycz, C. A., McNamara, J. R., \& Edwards, K. M. (2006). Women's risk perception and sexual victimization: A review of the literature. Aggression and Violent Behavior, 11, 441-456.

Green, D. M., \& Swets, J. A. (1966). Signal detection theory and psychophysics. Oxford, England: Wiley.

Hickman, S. E., \& Muehlenhard, C. L. (1997). College women's fears and precautionary behaviors relating to acquaintance rape and stranger rape. Psychology of Women Quarterly, 21, 527-547.

Koss, M. P., \& Gidycz, C. A. (1985). Sexual Experiences Survey: Reliability and validity. Journal of Consulting and Clinical Psychology, 53, $162-170$.

Koss, M. P., Gidycz, C. A., \& Wisniewski, N. (1987). The scope of rape: Incidence and prevalence of sexual aggression and victimization in a national sample of higher education students. Journal of Consulting and Clinical Psychology, 55, 162-170.

Loiselle, M., \& Fuqua, W. R. (2007). Alcohol's effects on women's risk detection in a date-rape vignette. Journal of American College Health, 55, 261-266.

Mason, G. E., Riger, S., \& Foley, L. A. (2004). The impact of past sexual experiences on attributions of responsibility for rape. Journal of Interpersonal Violence, 19, 1157-1171.

McCoy, S., Gibbons, F., Reis, T., Gerrard, M., Luus, E., \& Suffka, A. (1992). Perceptions of smoking risk as a function of smoking status. Journal of Behavioral Medicine, 15, 488-496.

Messman-Moore, T. L., \& Brown, A. L. (2006). Risk perception, rape, and sexual revictimization: A prospective study of college women. Psychology of Women Quarterly, 30, 159-172.

Messman-Moore, T. L., \& Long, P. J. (2003). The role of childhood sexual abuse sequelae in the sexual revictimization of women: An empirical review and theoretical reformulation. Clinical Psychology Review, 23, 537-571.

Muehlenhard, C. L., \& Linton, M. A. (1987). Date rape and sexual aggression in dating situations: Incidence and risk factors. Journal of Counseling Psychology, 34, 186-196.

Norris, J., Nurius, P. S., \& Dimeff, A. (1996). Through her eyes: Factors affecting women's perception of and resistance to acquaintance sexual aggression threat. Psychology of Women Quarterly, 20, 123-145.

Nurius, P. S., \& Norris, J. (1995). A cognitive ecological model of women's response to male sexual coercion in dating. Journal of Psychology and Human Sexuality, 8, 117-139.

Oishi, S., Lun, J., \& Sherman, G. D. (2007). Residential mobility, selfconcept, and positive affect in social interactions. Journal of Personality and Social Psychology, 93, 131-141.

Soler-Baillo, J. M., Marx, B. P., \& Sloan, D. M. (2005). The psychophysiological correlates of risk recognition among victims and non-victims of sexual assault. Behavior Research and Therapy, 43, 169-181.

Taylor, S. E., \& Brown, J. D. (1988). Illusion and well-being: A social psychological perspective on mental health. Psychological Bulletin, 103, 193-210.

Treat, T. A., Kruschke, J. K., Viken, R. J., \& McFall, R. M. (in press). Application of associative-learning paradigms to clinically relevant individual differences in cognitive processing. In Associative learning and conditioning: Human and animal applications. Oxford, United Kingdom: Oxford University Press.

Treat, T. A., McFall, R. M., Viken, R. J., \& Kruschke, J. K. (2001). Using cognitive science methods to assess the role of social information processing in sexually coercive behavior. Psychological Assessment, 13, $549-565$.

Treat, T. A., Viken, R. J., Kruschke, J. K., \& McFall, R. M. (2010). Role of attention, memory, and covariation-detection processes in clinically significant eating-disorder symptoms. Journal of Mathematical Psychology, 54, 184-195.

Turchik, K. A., Probst, D. R., Chau, M., Nigoff, A., \& Gidycz, C. A. (2007). Factors predicting the type of tactics used to resist sexual assault: A prospective study of college women. Journal of Consulting and Clinical Psychology, 75, 605-614.

Viken, R. J., Treat, T. A., Nosofsky, R. M., McFall, R. M., \& Palmeri, T. J. (2002). Modeling individual differences in perceptual and attentional processes related to bulimic symptoms. Journal of Abnormal Psychology, 111, 598-609.

Wilson, A. E., Calhoun, K. S., \& Bernat, J. A. (1999). Risk recognition and trauma-related symptoms among sexually revictimized women. Journal of Consulting and Clinical Psychology, 67, 705-710.

Yeater, E. A., McFall, R. M., \& Viken, R. J. (in press). The relationship between women's response effectiveness and a history of sexual victimization. Journal of Interpersonal Violence.

Yeater, E. A., \& O’Donohue, W. T. (2002). Sexual revictimization: The relationship among rape myths, risk perception, and ability to respond to high-risk situations. Journal of Interpersonal Violence, 17, 1135-1144.

Yeater, E. A., \& Viken, R. J. (in press). Factors affecting women's response choices to dating and social situations. Journal of Interpersonal Violence.

Yeater, E. A., Viken, R. J., Hoyt, T., \& Dolan, J. L. (2009). Self-other perspective and sexual attitudes affect estimates of sexual risk. Sex Roles, 61, 110-119.

Yeater, E. A., Viken, R. J., McFall, R. M., \& Wagner, L. R. (2006). Sexual attitudes and instructional set affect estimates of risk and response competence. Journal of Psychopathology and Behavioral Assessment, $28,233-242$.

Received January 27, 2009

Revision received February 1, 2010 Accepted February 2, 2010 